\title{
Microporous carbon spheres derived from resorcinol-formaldehyde solutions. A new approach to coat supports
}

\author{
Natalia Rey-Raap*, Sara F. Villanueva, J. Angel Menéndez and Ana Arenillas \\ Instituto Nacional del Carbón, CSIC, Apartado 73, 33080 Oviedo, Spain
}

\begin{abstract}
Microporous carbon spheres of different morphology and porosity were synthesized from resorcinol-formaldehyde solutions by a simple and fast procedure. Polymeric spheres were shaped by means of microwave heating. Carbonization and activation with carbon dioxide were then applied to obtain the intended final carbon spheres. The influence of the $\mathrm{pH}$, heating time and thermal treatments on the morphology and porosity of the carbon spheres was investigated. It was found that the size of the spheres, can be easily controlled during the synthesis process, specifically by modifying the $\mathrm{pH}$ of the precursor solution. An increase in the $\mathrm{pH}$ value from 3 to 5 led to carbon spheres with sizes of $4 \mu \mathrm{m}$ and $3.5 \mu \mathrm{m}$, respectively, whereas time seemed to have no effect. These results have been attributed to the chemical mechanisms of the polymerization reaction. On the other hand, microporosity was tailored during the thermal treatments. Carbon spheres with surface areas of $630 \mathrm{~m}^{2} / \mathrm{g}$ and $1500 \mathrm{~m}^{2} / \mathrm{g}$ were obtained by applying carbonization and physical activation, respectively. Furthermore, the synthesis method proposed allows to obtained liquid polymerized inks that can be further used to coat ceramic supports by a simple spray-drying process, which enhances the potential of these materials for several applications.
\end{abstract}

Keywords: carbon sphere, microwave-heating, spray-drying, coating

\section{Introduction}

Over the last few decades, there has been an explosive growth in the synthesis, characterization and application of carbon spheres, due to the fact that their size,

*Corresponding author. E-mail: nataliarey@fe.up.pt (present address: Laboratório Associado LSRE/LCM, Departamento de Engenharia Química, Faculdade de Engenharia, Universidade do Porto, R. Dr. Roberto Frias s/n, 4200-465 Porto, Portugal) 
morphology, porosity and chemical properties can be tailored for specific purposes. The final properties of carbon spheres are strongly influenced by the synthesis conditions under which they are prepared. Chemical vapour deposition (CVD), polymerization using templates, hydrothermal carbonization, inverse emulsion polymerization, extension of the Stöber method, etc. are some of the best-known examples of the wide list of methods studied. The reader is referred to a number of excellent reviews on these synthesis techniques, and on properties and applications of carbon spheres, which have already been published [1-5]. Another aspect worth bearing in mind, as it amends the final properties of carbon spheres, are the precursors employed, which comprise but are not limited to polymers, biomass-derived carbons, phenolic resins, benzene derivatives and other heterocyclic aromatic organic compounds $[2,4,6]$. Of these, phenolic resins have attracted most attention as carbon sphere precursors [7-17], and particularly resorcinol-formaldehyde (RF) precursor solutions which, as has been extensively reported, generally give rise to carbon gels [18-23].

A number of studies can be found in the literature focused on the use of RF solutions as precursors of carbon spheres, for the preparation of which several methods have been reported. The modified Stöber method has been one of the most employed techniques to prepare carbon spheres from RF solutions $[8,12,13,15,16]$. This method involves the use of additives such as ammonia, ethanol or templates, as well as long processing times. Another method that is widely used is that of inverse emulsion, where not only the use of additives is required but also a full control over the viscosity $[11,17]$. Recently, hydrothermal treatments has made it possible to synthesize carbon spheres easily without the need for additives [9]. However, this process is still tedious as long times are required. In order to reduce the processing time, ultrasounds and microwave radiation have been employed as suitable techniques to obtain carbon spheres $[7,10$, 14]. Although these methods allow the size and number of carbon spheres to be tailored via a rapid and easy route, the use of surfactants and full control of viscosity also seem to be essential requirements in these processes.

Leaving aside the above mentioned drawbacks, each of these techniques leads to carbon spheres with different final properties, and hence, the choice of the appropriate synthesis method will depend on the application for which the carbon spheres are intended. The widely-recognized advantages of RF solutions as carbon precursors (which include the possibility of tailoring their porous properties or of doping them with metals and 
heteroatoms $[11,24])$, confer on them great potential for preparing carbon spheres that offer optimum performances in a wide variety of applications, such as adsorption [1, 25], ultrafiltration [26], catalysis [16] or electrochemical systems [9, 13, 15, 17]. In some of these applications carbon spheres are used as coating of surfaces, nanoparticles or supports and, in these cases, spray-drying methods are preferable. This process involves spraying the precursor solution onto a support, which is then dried to give rise to the formation of polymeric spheres on its surface [1]. Finally, the polymer thus obtained is carbonized to yield the intended carbon spheres. In order to use the spraydrying method, liquid precursor solutions are needed, which rules out many of the aforementioned methods. Furthermore, the studies so far published on the spraying method, again refer to the need for additives [27-29]. Consequently, although several attempts to achieve a facile synthesis methodology for carbon spheres have been made, there is still an ongoing need for an alternative low-cost and easily scalable method to prepare liquid polymerized precursors that could be used in spray-drying processes for producing carbon spheres.

In the present work, microwave-assisted polymerization of resorcinol and formaldehyde was performed to obtain polymeric spheres, which were then transformed into carbon spheres. The novelty of the method proposed lies in the fact that i) the use of additives is avoided and ii) the synthesis time is greatly reduced. The process is thus simplified and become scalable. Furthermore, the method presented here has demonstrated to be an easy and suitable way to coat surfaces of ceramic solid catalysts, thereby extending the range of applications of carbon spheres prepared from RF precursor solutions.

\section{Experimental}

\subsection{Preparation of resorcinol-formaldehyde inks and flakes}

The selected starting materials were resorcinol (Indspec, 99\%), formaldehyde (Merck, 37 wt. $\%$ in water, stabilized by approx. 10 wt.\% of methanol), deionized water (produced by a Millipore water system) and a solution of sodium hydroxide $(\mathrm{NaOH} 5 \mathrm{M}$ solution prepared from solid $\mathrm{NaOH}$ (AnalaR Normapur, $99.9 \%$ ). 
Resorcinol was first dissolved in deionized water in an unsealed glass beaker under magnetic stirring. After dissolution, formaldehyde was added and the mixture was stirred until a homogeneous solution was obtained. Finally, the $\mathrm{pH}$ value was adjusted by adding the $\mathrm{NaOH}$ solution. The concentration of each reagent was selected on the basis of results previously reported. These concentrations are reported in the literature to be related to the $\mathrm{pH}$ of the precursor solution, the dilution ratio (D) and the molar ratio between the resorcinol and the formaldehyde $(\mathrm{R} / \mathrm{F})$ [30]. It should be highlighted that the dilution ratio refers to the molar ratio between the total solvent and the main reagents. Rey-Raap et al. provided an interesting illustration of the combinations of $\mathrm{pH}-$ D whereby the synthesis route via microwave heating did not lead to the formation of a solid material [18]. Based on that illustration, in the present work the precursor solutions were therefore prepared with $\mathrm{pH}$ values of 3 and 5 , using the stoichiometric $\mathrm{R} / \mathrm{F}$ molar ratio (i.e., 0.5) and a dilution ratio (D) fixed at 17.

Once prepared, each precursor solution was placed in a multimode microwave oven (inlab design and constructed [31]) at $85{ }^{\circ} \mathrm{C}$. The heating time was selected on the basis of previous experiments performed, in some of which microwave energy consumption was recorded as a function of time [10,19]. The time values selected were 2 and 3 hours to allow a polymeric ink (RF-ink) to be obtained instead of a wet-solid gel. Each polymeric RF-ink was air-dried at $85{ }^{\circ} \mathrm{C}$ for 3 hours until a solid thin-layer (i.e. flake) was obtained. These materials were labelled RFS, in reference to resorcinolformaldehyde spheres, followed by the $\mathrm{pH}$ value and the duration of the microwave heating in hours.

\subsection{Coating of the supports}

Coated supports were prepared by means of a spin-coating strategy based on the use of the precursor solution of resorcinol-formaldehyde gels prepared with a $\mathrm{pH}$ value of 3 and heated for 2 hours in the microwave oven. The supports used were monolithic catalyst substrates made of cordierite with a cell density of 200 cpsi and supplied by Corning Incorporated. Briefly, the supports were introduced into a glass flask containing $50 \mathrm{ml}$ of hot RF-ink for 3 minutes. The impregnated support was then centrifuged for 15 seconds at $600 \mathrm{rpm}$ and finally air-dried at $85{ }^{\circ} \mathrm{C}$ for $3 \mathrm{~h}$. In order to determine the optimum number of spin-coating cycles required, one and five cycles were performed. 


\subsection{Carbonization and physical activation}

The RFS samples and coated supports were heated for $2 \mathrm{~h}$ under a nitrogen atmosphere at $700{ }^{\circ} \mathrm{C}$ and under a $\mathrm{CO}_{2}$ atmosphere at $1000{ }^{\circ} \mathrm{C}$ in order to obtain carbonized (CS) and activated (ACS) carbon spheres, respectively.

\subsection{Sample characterization}

All the samples, RFS and supports (dried, carbonized and activated), were first outgassed at $0.1 \mathrm{mbar}$ and $120^{\circ} \mathrm{C}$ overnight in a Micromeritics VAcPrep 0.61 in order to remove humidity and other physisorbed gases. Their porous properties and morphology were then analyzed.

Nitrogen adsorption-desorption isotherm analysis were performed at $-196{ }^{\circ} \mathrm{C}$ using a Micromeritics Tristar 3020 instrument. The BET surface area $\left(\mathrm{S}_{\mathrm{BET}}\right)$ and micropore volume ( $\left.\mathrm{V}_{\text {DUB-N }}\right)$ of the samples were determined by applying the BET equation and the Dubinin-Radushkevic method to the $\mathrm{N}_{2}$ adsorption isotherms, respectively. Envelope pycnometry (Geopyc1360 envelope density analyzer from Micromeritics) was also employed to further analyze other porous properties such as bulk density and porosity. All samples were synthesized and characterized in duplicate to ensure reproducibility

The morphology of all the materials was examined using a Quanta FEG 650 scanning electron microscope. The samples were previously attached to an aluminum pin using conductive double-sided adhesive tape. An accelerating voltage of $25 \mathrm{kV}$ and a secondary electron detector EDT (Everhart-Thornley) were used in all the analyses. This technique was also employed to determine the size of the sphere. However, coulter analysis (LS13320 Beckman fitted with an ALM water module) was also used to verify these data. Coulter analysis were performed in triplicate.

\section{Results and discussion}

\subsection{Resorcinol-formaldehyde spheres (RFS)}


Regardless of the $\mathrm{pH}$ value of the precursor solution and the microwave heating time used to prepare the RF-inks, the results obtained from the $\mathrm{N}_{2}$ adsorption-desorption isotherms reveal that all the RFS samples are practically non-microporous materials $\left(\mathrm{S}_{\mathrm{BET}} \approx 1-2 \mathrm{~m}^{2} / \mathrm{g}\right)$. However, the results of the RF-inks studied also indicate that these materials have a bulk density of $0.6-0.8 \mathrm{~g} / \mathrm{cm}^{3}$ and a total porosity of $50 \%$, probably provided by pores of large size falling exclusively within the macroporosity range and, therefore, inappreciable under nitrogen adsorption analysis. These results are in good agreement with those obtained by SEM images, where large voids between spheres can be observed, as shown in Figure 1.
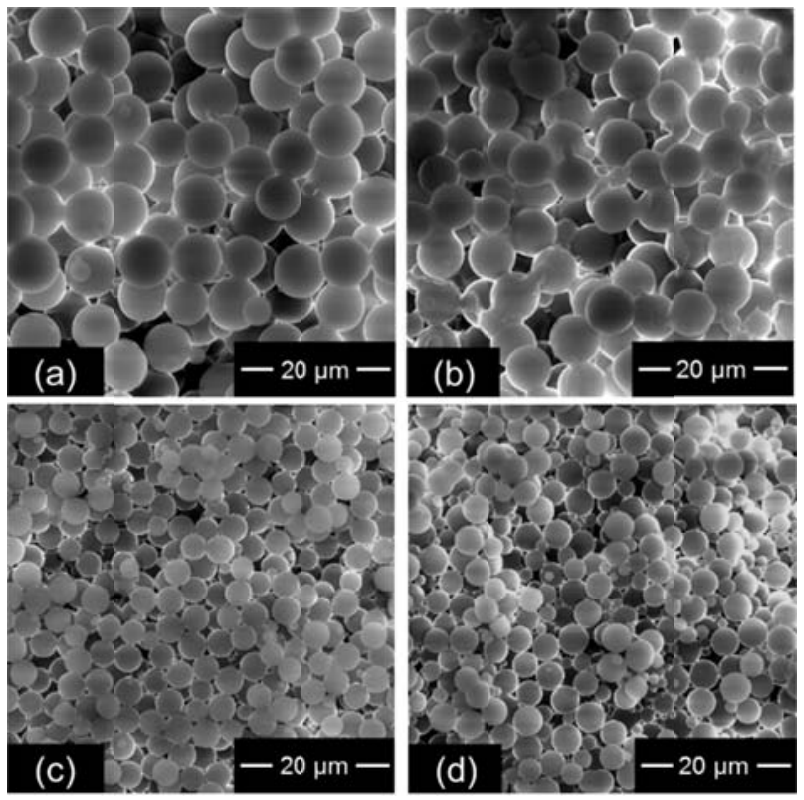

Figure 1. Scanning electron microscopy images of organic spheres labelled RFS in reference to resorcinol-formaldehyde spheres, followed by the $\mathrm{pH}$ value of the precursor solution and the duration of the microwave heating: (a) RSF-3-2h, (b) RSF-3-3h, (c) RSF-5-2h and (d) RSF-5-3h.

The size of the spheres is markedly influenced by the initial $\mathrm{pH}$ of the precursor solution, whereas time seems to have no effect (Figure 1). Whatever the time selected, an increase in the $\mathrm{pH}$ value leads to spheres with sizes ranging from 6-7 $\mu \mathrm{m}$ for a $\mathrm{pH}$ value of 3 (samples RFS-3-2h and RFS-3-3h) to 5-6 $\mu \mathrm{m}$ when the $\mathrm{pH}$ is fixed at 5 (samples RFS-5-2h and RFS-5-3h). These results can be attributed to the polymerization reaction between resorcinol and formaldehyde, which involves two reactions: i) the addition of formaldehyde to resorcinol and ii) the subsequent condensation of the hydroxymethyl derivatives formed (Figure 2). 


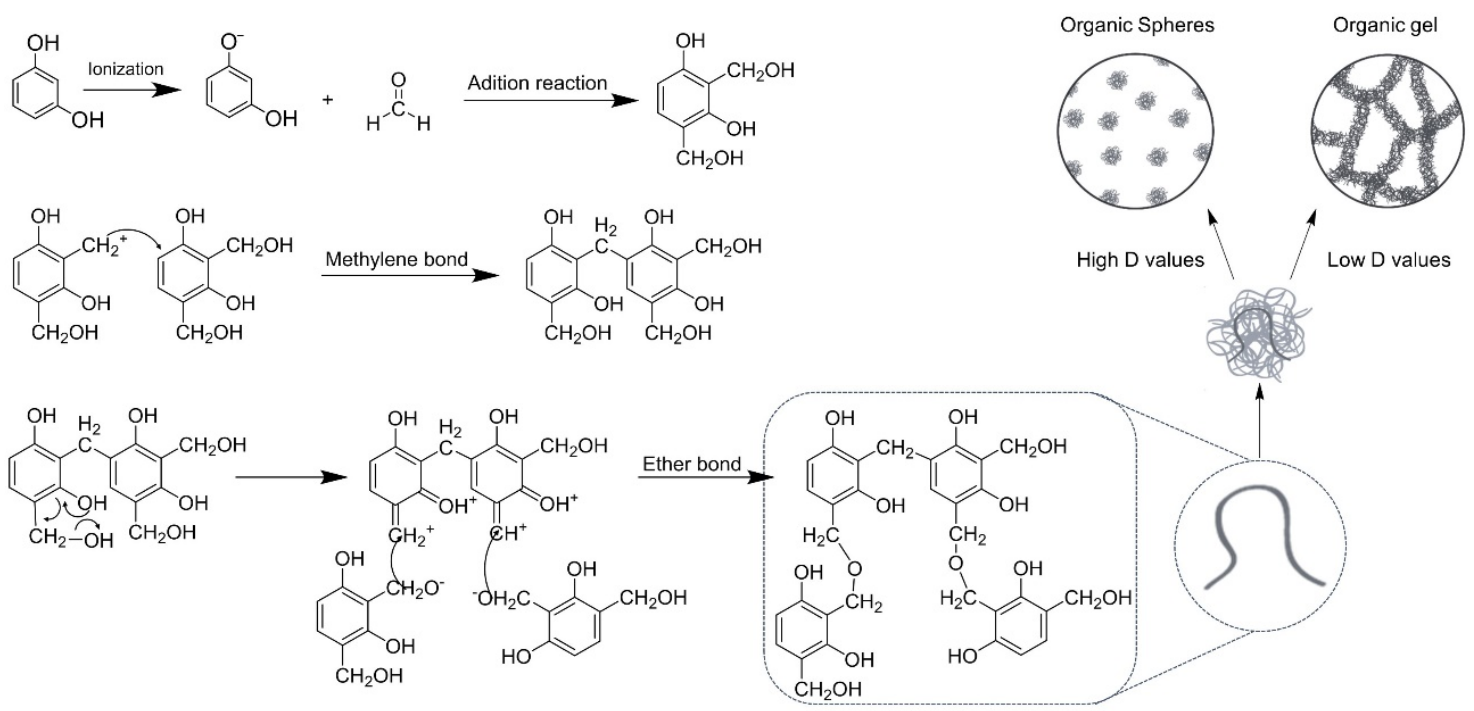

Figure 2. Mechanism for the polymerization reaction of resorcinol with formaldehyde to form organic spheres.

Initially, resorcinol anions are formed due to the abstraction of hydroxyl hydrogens, which generally allows the addition of formaldehyde in positions 2 and 4 (addition reaction) [30]. At the same time, the hydroxymethyl derivatives lose $\mathrm{OH}$ groups to form benzyl-type cations (condensation reaction). Each cation reacts with a benzene ring of another molecule giving rise to methylene and ether bonds. As the reaction proceeds, the number of bonds between the rings increases to form the polymer backbone, which leads to cross-linked polymer clusters, also named primary particles [18, 24]. Unlike in the synthesis of organic gels, these primary particles do not aggregate and crosslink with each other due to the high D value used to prepare the precursor solutions. Indeed, the large amount of water (high dilution ratio value, $\mathrm{D}=17$ ) increases the distance between primary particles, and hence, prevents the solution from reaching the gelation point [30]. Therefore, isolated organic spheres are formed, i.e. RF-inks (Figure 3a) instead of organic wet-solid gels (Figure $3 b$ and $3 d$ ) are yielded. Once the RF-inks have been prepared, any excess solvent is removed. Solid materials composed exclusively of quasi non-interconnected micro-spheres, like those shown in Figure 3c, are then obtained. 

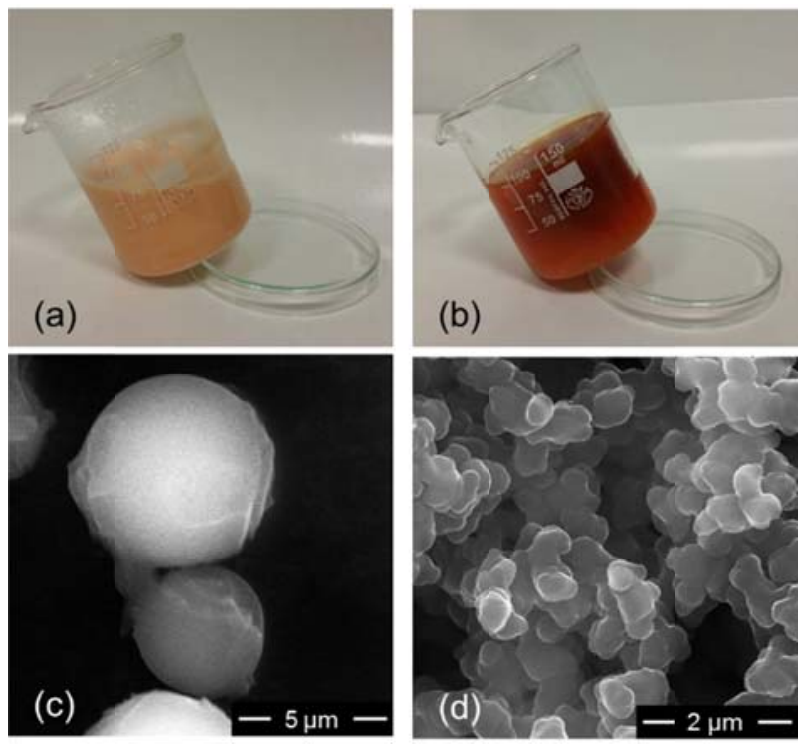

Figure 3. Pictures of the resorcinol-formaldehyde ink (a) and a wet-solid gel (b) and SEM images of the RF spheres (c) and a carbon gel (d).

The polymerization reaction shown in Figure 2 may be catalyzed by basic or acid agents, which accelerates the addition reaction $[24,30]$. In the present study, the precursor solutions prepared had an initial $\mathrm{pH}$ value of 3 , so a basic agent (a $\mathrm{NaOH}$ solution) was added to some samples, to increase the initial $\mathrm{pH}$ value to 5 . The appearance of hydroxymethyl derivatives is favored at high $\mathrm{pH}$ values via the deprotonation of resorcinol. As a consequence, the addition reaction is favored by increasing the $\mathrm{pH}$, and this results in the formation of spheres of small size, as shown in Figure 1. Therefore, it can be said that the RF-inks prepared lead to materials made of polymeric spheres of different sizes, obtained by selecting the appropriate concentration of reagents, specially $\mathrm{pH}$ and $\mathrm{D}$. These RF spheres can be easily be transformed into carbon spheres by means of suitable thermal treatments such as carbonization or activation processes. These thermal post-treatments will provide carbon spheres with thermal stability and porosity.

\subsection{Carbonized and activated spheres}

The effect of the thermal treatments on the morphology and porosity of the carbon spheres was evaluated. As expected, the effect of the $\mathrm{pH}$ and microwave heating time on the size of the carbon spheres was similar to that of the organic samples, i.e. time has no effect while the size of the spheres decreases with $\mathrm{pH}$, as can be seen by comparing samples CS-3-2h and CS-5-2h, in Figure $4 \mathrm{a}$ and 4b, respectively. 

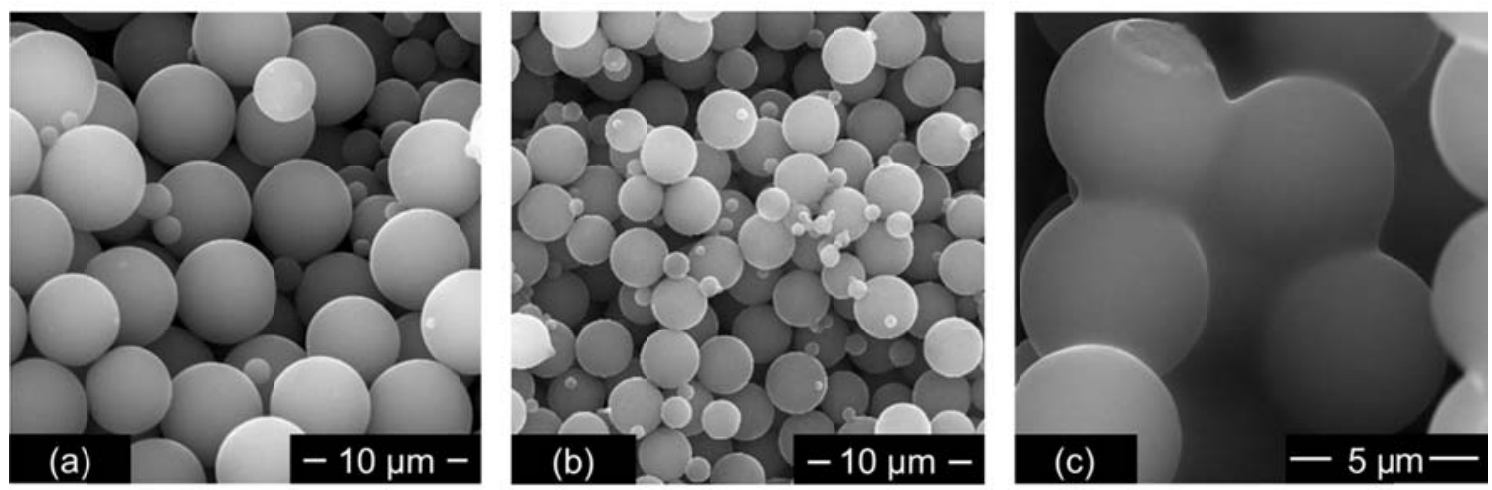

Figure 4. Scanning electron microscopy images of carbon spheres labelled CS, followed by the $\mathrm{pH}$ value of the precursor solution and the duration of the microwave heating: (a) CS-3-2h and (b) CS-5-2h; and (c) SEM image of activated spheres AS-3-2h.

The size of the carbon spheres decreases slightly during the carbonization process, as inferred by comparing Figure 1 and 4 . The size of the spheres decreases from 6-7 $\mu \mathrm{m}$ to around a mean value of $4 \mu \mathrm{m}$ and from $5-6 \mu \mathrm{m}$ to around $3.5 \mu \mathrm{m}$ for samples with a $\mathrm{pH}$ value of 3 and 5, respectively. These results are in agreement with those obtained by coulter analysis of the carbonized spheres, shown in Figure S1, and with that of carbon spheres prepared via more complex processes [11, 17], which underscores the relevance of the method proposed in the present work. Nonetheless, it is also important to highlight that some studies also show that it is possible to prepared carbon spheres of smaller sizes, which might be an advantage for some applications. However, the methods to obtain those smaller spheres require solvents [7, 13, 15], long synthesis times $[12,15,16]$ and even surfactants $[14,16]$, which hinders their industrial implementation and increases their production cost.

The decrease in the size of the carbon spheres can be attributed to the release of volatile matter, which occurs mainly above $500{ }^{\circ} \mathrm{C}$ [24], causing a slight shrinkage of the polymeric spheres. This process also contributes to the formation of micropores, as previously reported in literature $[12,21]$. Furthermore, in addition to the carbonization effects, the activation process leads to a certain degree of merging between spheres. Organic spheres are composed of primary particles with a great amount of surface oxygenated groups [23]. It has been widely demonstrated that polymers, especially those with oxygenate groups in their backbone structure such as ethers, have strong interactions with $\mathrm{CO}_{2}$ that lead to certain degree of sintering of the spheres [32]. 
Therefore, the contact of the organic spheres with $\mathrm{CO}_{2}$ at high temperatures, gives rise to the merging effect shown in Figure 4c.

The carbonization and activation processes also gave rise to differences in porosity. As can be seen from Figure 5, the RF spheres are non-porous, while the isotherms of samples CS and ACS are of type I according to the International Union of Pure and Applied Chemistry (IU-PAC) classification, which corresponds to microporous materials. The thermal treatments involve the breaking of $\mathrm{C}-\mathrm{O}$ bonds and $\mathrm{C}-\mathrm{H}$ bonds at temperatures of $350{ }^{\circ} \mathrm{C}$ and $550{ }^{\circ} \mathrm{C}$, respectively [21]. The breakage of bonds not only leads to the rearrangement of molecules, resulting in a more stable structure, but also to the generation of small pores inside and on the surface of the spheres. Thereafter, as expected, samples CS and ACS exhibit greater microporosity than sample RFS. Moreover, in sample ACS a larger volume of nitrogen is adsorbed at low relative pressures than in sample CS, which indicates the presence of a larger volume of micropores $\left(\mathrm{S}_{\mathrm{BET}}\right.$ increases from $630 \mathrm{~m}^{2} / \mathrm{g}$ to $1500 \mathrm{~m}^{2} / \mathrm{g}$ and $\mathrm{V}_{\text {DUB }}$ from 0.24 to 0.61 $\mathrm{cm}^{3} / \mathrm{g}$ ). $\mathrm{CO}_{2}$ reacts with the active sites on the carbon surface via an oxidation reaction, which results in oxygen complexes that are unstable at high temperatures $\left(800-950{ }^{\circ} \mathrm{C}\right)$ and thus, decompose, leading to the generation of greater microporosity [22]. These values are similar to those obtained for carbon and activated xerogels prepared from RF precursor solutions [19-22], which means that the effect of these thermal treatments are identical in both types of materials: RF spheres and xerogels.

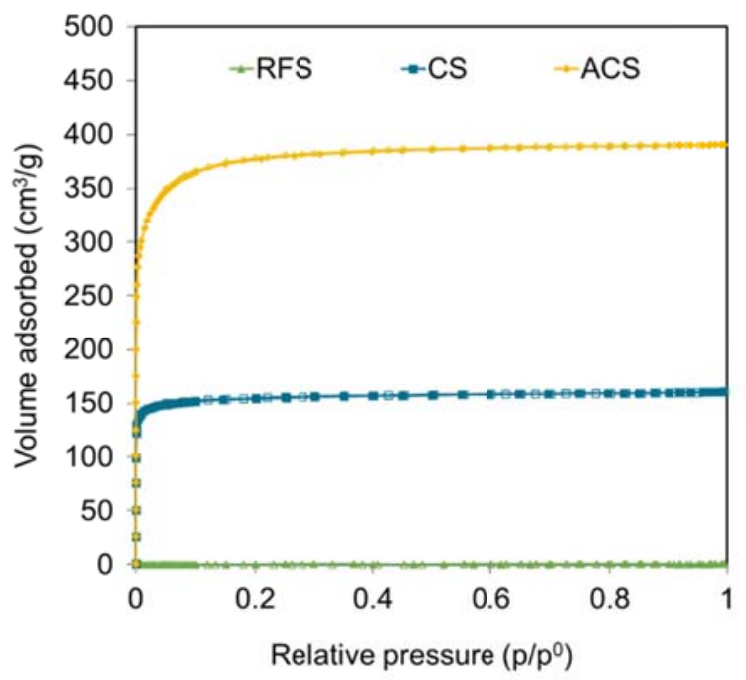


Figure 5. Nitrogen adsorption-desorption isotherms of resorcinol-formaldehyde spheres (RSF), carbon spheres (CS) and activated spheres (ACS) prepared from a precursor solution with a $\mathrm{pH}$ value of 3.0 and a duration of the microwave heating of $2 \mathrm{~h}$.

Alike organic gels, RF-flakes can be easily manipulated and exposed to thermal treatment as explained above. The RFS sample can also be stored and transported in its dry form (Figure 6) or used to make a suspension for use as coating, with the saving of both storage space and transport costs.

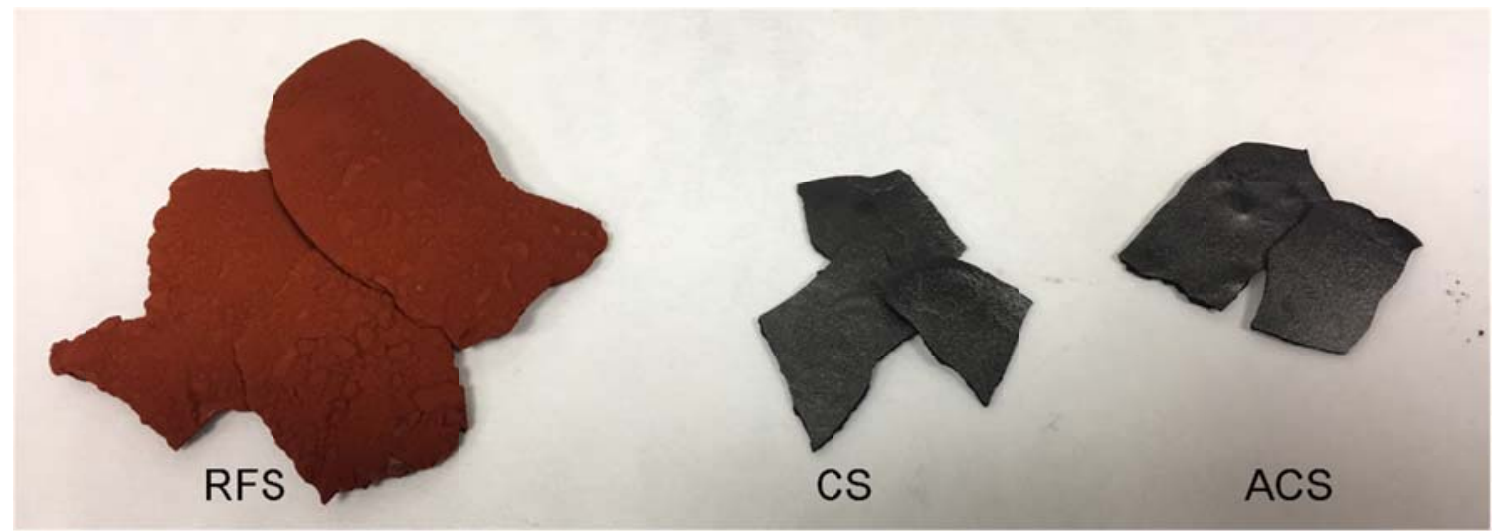

Figure 6. Resorcinol-formaldehyde flakes composed of (a) organic spheres and its (b) carbonized (CS) and (c) activated (ACS) counterpart.

\subsection{Coated supports}

From the results reported above, it can be inferred that the size of the spheres can be easily controlled during the synthesis process, while microporosity is tailored during the thermal post-treatments. The properties obtained would be particularly useful in applications such as $\mathrm{CO}_{2}$ adsorption [5] or molecular sieves [22]. It is therefore of great interest to study if these materials can be used as coatings. To this end, two supports made of cordierite were spin-coated by means of one and five cycles, respectively and by using a RF-ink prepared from a precursor solution with a $\mathrm{pH}$ value of 3 which was then microwave-heated for $2 \mathrm{~h}$. This sample was selected because of its higher degree of homogeneity, shown in Figure 4. Figure 7 shows the SEM images obtained in which important differences can be observed. It can be seen that one cycle spin-coating is not enough to cover all the surfaces of the supports or the most inner parts (Figure 7a). However, an increase in the number of cycles to five, results in a well-coated support with several layers of spheres (Figure 7b). The RF spheres obtained are similar to those 
without any support (Figure 1), demonstrating that, during the elimination of excess solvent, the morphology of the spheres is not modified by the support.
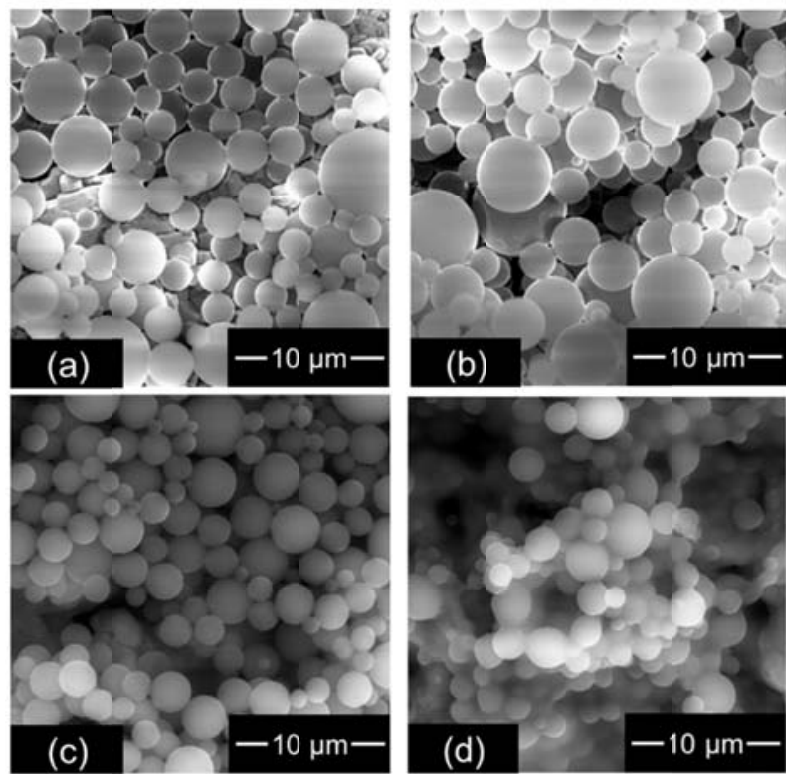

Figure 7. Scanning electron microscopy images of supports coated with polymeric spheres (sample RFS-3-2h) prepared by one (a) and five spin-coating cycles (b) and of the carbonized (c) and activated (d) coating.

By further carbonizing or activating the coated support, a microporous layer composed of carbon micron-spheres is obtained, as shown in Figure $7 \mathrm{c}$ and $7 \mathrm{~d}$, respectively. These samples exhibit a homogenous coating of carbon spheres with different degrees of microporosity, which make them useful for numerous applications, such as $\mathrm{CO}_{2}$ adsorption [5, 6] and energy storage [9, 13, 15]. In the supplementary information a video is provided, which shows how inks can be manipulated and the different possible ways of coating surfaces.

\section{Conclusions}

Resorcinol-formaldehyde (RF) solutions were used as precursors of RF-spheres, which were then carbonized and activated to obtained carbon spheres. The results obtained demonstrate that the size of the spheres can be easily controlled during the synthesis process, while microporosity is tailored during thermal post-treatments. These results are in agreement with the finding of studies on the growth of clusters and the tailoring of the porosity of RF and carbon gels, which provides a valuable contribution to materials science, since the exhaustive knowledge about the synthesis of carbon gels 
can be applied to the production of carbon spheres with tailored chemical and porous properties by means of microwave heating.

Furthermore, the process proposed in the present study allows the preparation of carbon spheres with a controlled morphology and porosity via a simple and easily scalable method. The time required to prepare carbon spheres is shorter in comparison with previous methods reported in literature. The synthesis method proposed also yields RF inks with a fluidity suitable for coating different supports, not only by impregnation but also by spraying methods. These results evidence the significance of the method as this coating procedure can be applied to a wide range of supports regardless of their shape. Accordingly, it is to be hoped that the use of carbon spheres increases in the near future, taking advantage of the improvements developed in the presented work.

\section{Acknowledgements}

Financial support from the Ministerio de Economía y Competitividad of Spain, under Project CTQ2014-54772-P is greatly acknowledged.

\section{References}

[1] C. Moreno-Castilla, Colloidal and micro-carbon spheres derived from lowtemperature polymerization reactions, Adv. Colloid Interface Sci., 236 (2016) 113-141.

[2] J. Liu, N.P. Wickramaratne, S.Z. Qiao, M. Jaroniec, Molecular-based design and emerging applications of nanoporous carbon spheres, Nat. Mater., 14 (2015) 763-774.

[3] A.A. Deshmukh, S.D. Mhlanga, N.J. Coville, Carbon spheres, Mater. Sci. Eng., R, 70 (2010) 1-28.

[4] A.D. Roberts, X. Li, H. Zhang, Porous carbon spheres and monoliths: morphology control, pore size tuning and their applications as Li-ion battery anode materials, Chemical Society Reviews, 43 (2014) 4341-4356.

[5] P. Zhang, Z.A. Qiao, S. Dai, Recent advances in carbon nanospheres: Synthetic routes and applications, Chem. Commun., 51 (2015) 9246-9256.

[6] L. Liu, Z.-H. Xie, Q.-F. Deng, X.-X. Hou, Z.-Y. Yuan, One-pot carbonization enrichment of nitrogen in microporous carbon spheres for efficient $\mathrm{CO} 2$ capture, $\mathrm{J}$ Mater Chem A, 5 (2017) 418-425.

[7] V.G. Pol, L.K. Shrestha, K. Ariga, Tunable, functional carbon spheres derived from rapid synthesis of resorcinol-formaldehyde resins, ACS Appl. Mater. Interfaces, 6 (2014) 10649-10655. 
[8] A.H. Lu, G.P. Hao, Q. Sun, Can carbon spheres be created through the stöber method?, Angew. Chem. Int. Ed., 50 (2011) 9023-9025.

[9] Y. Choi, Y. Kim, K.Y. Kang, J.S. Lee, A composite electrolyte membrane containing high-content sulfonated carbon spheres for proton exchange membrane fuel cells, Carbon, 49 (2011) 1367-1373.

[10] J.A. Menéndez, E.J. Juárez-Pérez, E. Ruisánchez, E.G. Calvo, A. Arenillas, A microwave-based method for the synthesis of carbon xerogel spheres, Carbon, 50 (2012) 3555-3560.

[11] Z. Zapata-Benabithe, J. De Vicente, F. Carrasco-Marín, C. Moreno-Castilla, Synthesis, surface characteristics, and electrochemical capacitance of $\mathrm{Cu}$-doped carbon xerogel microspheres, Carbon, 55 (2013) 260-268.

[12] C. Zhang, K.B. Hatzell, M. Boota, B. Dyatkin, M. Beidaghi, D. Long, W. Qiao, E.C. Kumbur, Y. Gogotsi, Highly porous carbon spheres for electrochemical capacitors and capacitive flowable suspension electrodes, Carbon, 77 (2014) 155-164.

[13] J.H. Zhang, J.Y. Feng, T. Zhu, Z.L. Liu, Q.Y. Li, S.Z. Chen, C.W. Xu, Pd-doped Urchin-like MnO2-carbon Sphere Three-dimensional (3D) Material for Oxygen Evolution Reaction, Electrochim. Acta, 196 (2016) 661-669.

[14] N. Tonanon, W. Intarapanya, W. Tanthapanichakoon, H. Nishihara, S.R. Mukai, H. Tamon, Submicron mesoporous carbon spheres by ultrasonic emulsification, J. Porous Mater., 15 (2008) 265-270.

[15] Y. Wang, B. Chang, D. Guan, X. Dong, Mesoporous activated carbon spheres derived from resorcinol-formaldehyde resin with high performance for supercapacitors, J. Solid State Electrochem., 19 (2015) 1783-1791.

[16] B. Chang, Y. Li, Y. Guo, B. Yang, Simple fabrication of magnetically separable mesoporous carbon sphere with excellent catalytic performance for biodiesel production, J. Taiwan Inst. Chem. Eng., 60 (2016) 241-246.

[17] Z. Zapata-Benabithe, F. Carrasco-Marín, J. De Vicente, C. Moreno-Castilla, Carbon xerogel microspheres and monoliths from resorcinol-formaldehyde mixtures with varying dilution ratios: Preparation, surface characteristics, and electrochemical double-layer capacitances, Langmuir, 29 (2013) 6166-6173.

[18] N. Rey-Raap, J.A. Menéndez, A. Arenillas, RF xerogels with tailored porosity over the entire nanoscale, Micropor. Mesopor. Mater., 195 (2014) 266-275.

[19] N. Rey-Raap, J.A. Menéndez, A. Arenillas, Optimization of the process variables in the microwave-induced synthesis of carbon xerogels, J. Sol-Gel Sci. Technol., (2013) $1-10$.

[20] N. Rey-Raap, A. Arenillas, J.A. Menéndez, A visual validation of the combined effect of $\mathrm{pH}$ and dilution on the porosity of carbon xerogels, Micropor. Mesopor. Mater., 223 (2016) 89-93. 
[21] C. Lin, J.A. Ritter, Carbonization and activation of sol-gel derived carbon xerogels, Carbon, 38 (2000) 849-861.

[22] J.F. Vivo-Vilches, F. Carrasco-Marín, A.F. Pérez-Cadenas, F.J. Maldonado-Hódar, Fitting the porosity of carbon xerogel by $\mathrm{CO} 2$ activation to improve the TMP/n-octane separation, Micropor. Mesopor. Mater., 209 (2015) 10-17.

[23] I.D. Alonso-Buenaposada, L. Garrido, M.A. Montes-Morán, J.A. Menéndez, A. Arenillas, An underrated variable essential for tailoring the structure of xerogel: the methanol content of commercial formaldehyde solutions, J. Sol-Gel Sci. Technol., (2017) 1-11.

[24] C. Moreno-Castilla, F.J. Maldonado-Hódar, Carbon aerogels for catalysis applications: An overview, Carbon, 43 (2005) 455-465.

[25] M.V. Galaburda, V.M. Bogatyrov, J. Skubiszewska-Zięba, O.I. Oranska, D. Sternik, V.M. Gun'ko, Synthesis and structural features of resorcinol-formaldehyde resin chars containing nickel nanoparticles, Appl. Surf. Sci., 360, Part B (2016) 722730 .

[26] G. Qin, J. He, W. Wei, Ultrafiltration Carbon Membranes from Organic Sol-Gel Process, Chem. Eng. Commun., 203 (2016) 381-388.

[27] Y. Yan, F. Zhang, Y. Meng, B. Tu, D. Zhao, One-step synthesis of ordered mesoporous carbonaceous spheres by an aerosol-assisted self-assembly, Chem. Commun., (2007) 2867-2869.

[28] J. Zhou, Y. Wang, J. Wang, W. Qiao, D. Long, L. Ling, Effective removal of hexavalent chromium from aqueous solutions by adsorption on mesoporous carbon microspheres, J. Colloid Interface Sci., 462 (2016) 200-207.

[29] X. Li, J. Zhou, J. wang, W. Qiao, L. Ling, D. Long, Large-scale synthesis of mesoporous carbon microspheres with controllable structure and nitrogen doping using a spray drying method, RSC Adv., 4 (2014) 62662-62665.

[30] N. Rey-Raap, A. Arenillas, J.A. Menéndez, Formaldehyde in the Synthesis of Resorcinol-Formaldehyde Carbon Gels in: A. Patton (Ed.) Formaldehyde: Synthesis, Applications and Potential Health Effects, Nova Science2015.

[31] J.M. Bermúdez, D. Beneroso, N. Rey-Raap, A. Arenillas, J.A. Menéndez, Energy consumption estimation in the scaling-up of microwave heating processes, Chem Eng Process, 95 (2015) 1-8.

[32] M. Bhamidipati, B. Sridharan, A.M. Scurto, M.S. Detamore, Subcritical CO2 sintering of microspheres of different polymeric materials to fabricate scaffolds for tissue engineering, Materials Science and Engineering: C, 33 (2013) 4892-4899. 
Microporous carbon spheres derived from resorcinol-formaldehyde solutions. A new approach to coat supports

Natalia Rey-Raap*, Sara F. Villanueva, J. Angel Menéndez and Ana Arenillas Instituto Nacional del Carbón, CSIC, Apartado 73, 33080 Oviedo, Spain

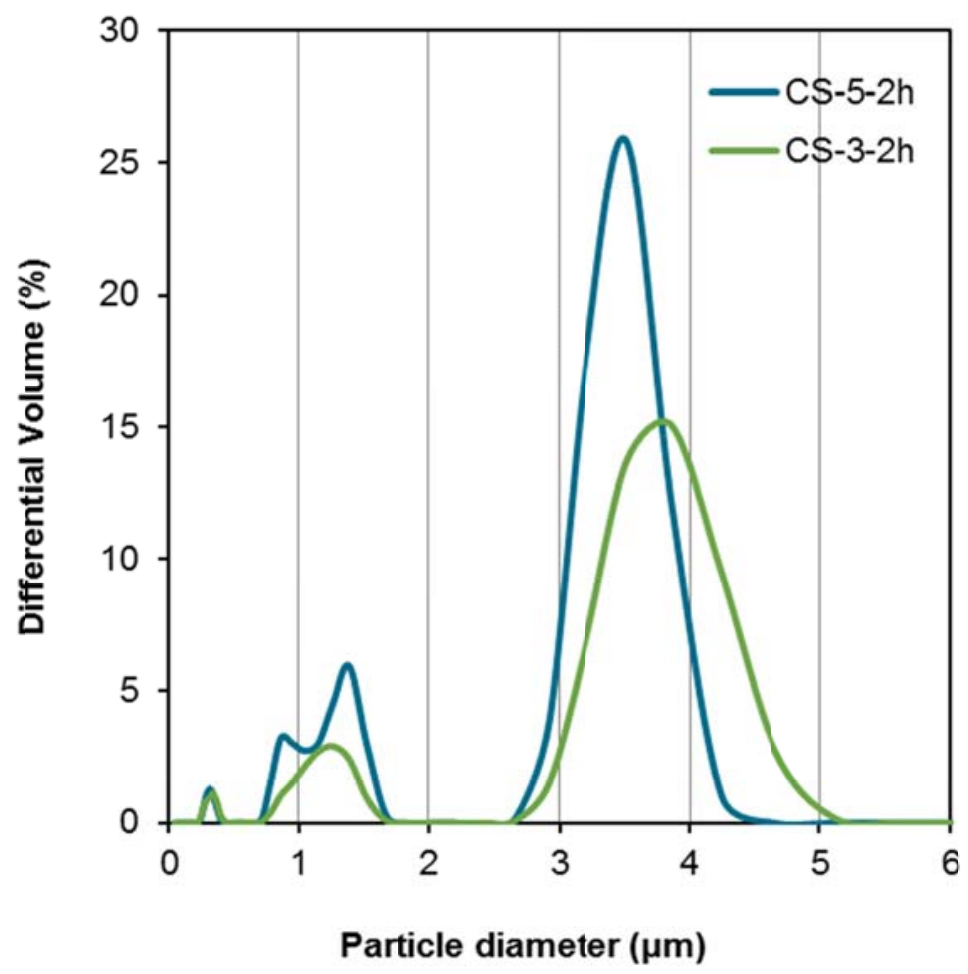

Figure S1. Carbon sphere size distribution measured by coulter counter analysis. 